\title{
Reprocessing of injection-molded magnetorheological elastomers based on TPE matrix
}

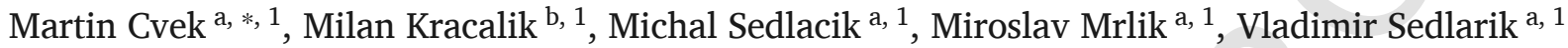 \\ ${ }^{a}$ Centre of Polymer Systems, University Institute, Tomas Bata University in Zlín, Trida T. Bati 5678, 760 01, Zlín, Czech Republic \\ ${ }^{\mathrm{b}}$ Johannes Kepler University Linz, Institute of Polymer Science, Altenberger Straße 69, 4040, Linz, Austria
}

\section{A R T I C L E I N F O}

Keywords:

Smart materials

Mechanical testing

Injection molding

Recycling

Thermoplastic elastomer

\begin{abstract}
A B S T R A C T
Nowadays, the magnetorheological elastomers (MREs) represent an important composite group with a wide range of applications. They are however predominantly typified by chemically cross-linked polymer matrices which makes them difficult to be reprocessed or recycled. Here, we demonstrate the concept of the MREs' reprocessing for the first time. The thermoplastic elastomer (TPE) was adopted as a suitable matrix allowing the MRE production via injection-molding, while making them also reusable. Each processing iteration was accompanied by thermo-mechanical degradation causing the gradual TPE oxidation, decrease in the TPE molecular weight and a viscosity reduction of their melts. In the MREs, the unexpected processing-induced particle/matrix bonding was revealed, which promoted their stiffening. As a result, the magnetic field-induced particle mobility was limited decreasing the magnetorheological activity of the MREs by tens of percent per the processing cycle. We expect that the injection-molded TPE-based MREs could offer a new pathway for producing the smart engineering composites owing to the ability to be easily reprocessed.
\end{abstract}

\section{Introduction}

Smart composite materials containing magnetically-permeable micro-particles dispersed in an elastomeric matrix are well-known as magnetorheological (MR) elastomers (MREs). These systems are characterized by their precise control of viscoelastic moduli once they are exposed to defined external magnetic fields. In recent years, the MREs have been a subject of numerous studies showing the enormous potential in civil engineering [1], automotive [2], medical [3,4] or military fields [5]. In this sense, they must meet certain criteria in order to reliably work under specific operating conditions. To design high-performance MREs, the aspects related to magnetic particles such as their inherent magnetic properties, the average size, or spatial distribution should be considered [6]. Besides that, the matrix elasticity plays an essential role in achieving high MR performance as shown by Chertovich et al. [7].

To this date, the vast majority of MREs are fabricated based on chemically cross-linked matrices including natural rubber [8,9] as well as their synthetic alternatives, e.g. silicone $[5,10]$, cis-polybutadiene [11,12], or nitrile [13] rubbers. Also polyurethanes (PURs) either in their raw form [14] or with plasticizers added [15] have been used as the MRE matrix material. Being thermosetting polymers, the reprocessing of MREs based on rubbers or PURs is practically infeasible.

Currently, traditional materials are being replaced by their modern analogues which also applies to MREs. Due to an increasing number of MR devices, the recycling of these systems may become a critical aspect in the next decades in view of preventing contamination of the environment with polymer wastes. Recently, the first attempts to economize MREs' fabrication appeared when Ubaidillah et al [16]. used a waste tire material as a matrix for MREs. In their study, crumb rubber was mixed with magnetic particles, but also a vulcanizing system containing sulfur, zinc oxide, and stearic acid was added. The final product thus exhibited high zero-field modulus, most probably due to high cross-link density, and the possibilities of its reprocessing were not further studied. It can be assumed that the fabrication of MREs based on matrices that can be easily reprocessed is the most reasonable choice from an environmental point of view.

Thermoplastic elastomers (TPEs) are special polymeric materials that can be considered as a desirable alternative to conventional vulcanized rubbers. Besides their high elasticity, they can be processed at elevated temperatures as conventional thermoplastics [17]. Thus, TPEs have the ability to be melt-processed, reused, and recycled unlike their chemically cross-linked analogues, i.e. rubbers, which makes them suit-

\footnotetext{
* Corresponding author.

Email address: cvek@utb.cz (M. Cvek)

$1 \%$ contribution made by each author in producing manuscript: Martin Cvek 65\%, Milan Kracalik 5\%, Michal Sedlacik 10\%, Miroslav Mrlik 10\%, Vladimir Sedlarik 10\%.
} 
able candidates for the development of "green" MREs. These features stem from their microstructure that contains physical cross-links between hard segments with either a high melting temperature or high glass transition temperature $\left(T_{g}\right)$, and soft segments with a low $T_{g}$. The soft segments elongate upon strain-induced deformation, while the hard ones represent anchoring points needed for elastic recovery $[17,18]$. To this date, the research background devoted to the development of MREs based on the TPE matrix is rather limited [19-22].

In the previous studies, the effects of silica coating $[19,23]$, processing conditions [24] or particle concentration [13,20,24,25] on the MREs' properties were investigated, however the aspects of their reprocessing remained omitted. Further, the production of MREs was previously ensured mainly via facile techniques such as casting $([5,10,26]$ among others), or compression molding [6,19].

At this point it should be emphasized that an immense advantage of TPEs is their capability to be manufactured via injection-molding (IM). This technique allows higher production rates and gives the possibility to fabricate elements with detailed features and complex geometries, which is difficult to achieve with the conventional production methods. Using this production technique, Volpe et al. [27] recently fabricated the anisotropic magneto-sensitive composites based on ethylene vinyl acetate. They used a custom-built aluminum mold with the accommodated electromagnet to produce the magnetic field during the filling process resulting in the particle alignment and consequently higher tensile modulus of as-prepared composites. Moreover, they were able to change the orientation of the field with respect to the sample axis tilting the particle chains in the composites [28]. The same research group further succeeded with the preparation of magnetic foams via foam injection-molding, which gives access to low-density magnetic composites [29].

In a view of cited papers, it becomes clear that some aspects, especially related to the MRE reprocessing, have not been covered in the existing literature so far. In this work, two main domains can be distinguished. In order to prevent environmental contamination with polymer wastes and to economize both, raw material consumption and energy resources, the fabrication and also reprocessing of TPE-based MREs were studied in detail. The composites were fabricated via the IM technique using a homogeneous mixture of carbonyl iron (CI) particles and TPE matrix, and they were further subjected to three reprocessing cycles. The effects of multi-processing on the molecular, optical, structural, viscoelastic and the MR properties of TPE-based MREs was complexly investigated and discussed.

\section{Experimental}

\subsection{Materials}

Propylene-based thermoplastic elastomer (trade name Vistamaxx ${ }^{\mathrm{TM}}$ 6202, ExxonMobil, USA) is primarily composed of isotactic propylene (85 wt $\%)$ repeat units with random ethylene $(15 \mathrm{wt} \%)$ distribution produced using metallocene catalyst technology. Vistamaxx (melt flow index of $7.4 \mathrm{~g} / 10 \mathrm{~min}$, with a density of $0.861 \mathrm{~g} \mathrm{~cm}^{-3}$ ) was selected as a suitable matrix material for the MREs' preparation due to its good elasticity, easy processability, excellent chemical resistance and long-term aging. The carbonyl iron (CI) particles (CN grade, iron content minimum of $99.5 \%, \mathrm{~d}_{50}=6.5-8.0 \mu \mathrm{m}$ ) supplied by BASF (Germany) were used as a magnetic micro-filler. Tetrahydrofuran (THF, purity of $\geq 99.9 \%$, inhibitor-free) sourced from Sigma-Aldrich (USA) was used as a dissolving medium in the chromatography investigations.

\subsection{MREs' fabrication and reprocessing}

The homogeneous polymer blends of the CI particles and the TPE were prepared by mixing both components on a twin-screw counter-ro- tating mixer provided by Brabender (Duisburg, Germany) with a volume capacity of $\sim 50 \mathrm{~cm}^{3}$. The instrument was heated at an operating temperature of $185^{\circ} \mathrm{C}$ (all heating zones), while the feeding time was set to $1 \mathrm{~min}$ followed by $4 \mathrm{~min}$ of compounding with a rotor speed at $50 \mathrm{rpm}$. The mixing process was performed for the CI-filled TPE (MREs) as well as for the neat TPEs in order to investigate the effects of processing on neat matrix degradation. In the case of the MREs, the TPE pellets were dosed firstly with the subsequent addition of the calculated amount of the CI particles creating a mixture of $80 \mathrm{wt} \%$ ( $~ 31$ vol\%) particle concentration. The mixtures were left to cool down to the laboratory temperature and cut into small pieces, and further used in the IM processing. This process was performed using a HAAKE MiniJet Pro - Piston IM System (Thermo Scientific, Germany) to prepare the disc-shaped specimens of $25 \mathrm{~mm}$ diameter, and $1.25 \mathrm{~mm}$ height. The optimal parameters for the IM included the temperature of the cylinder $\left(185^{\circ} \mathrm{C}\right)$, temperature of the mold $\left(35^{\circ} \mathrm{C}\right)$, the injection pressure/time (260 bar, $2 \mathrm{~s}$ ), and post-pressure/time (70 bar, $3 \mathrm{~s}$ ). As-prepared product was considered as a virgin material (R0). After its testing and characterization, the sample was then cut into small pieces, and the whole batch was reprocessed (mixing and injection-molding) under the same conditions as the virgin material (Fig. 1). The reprocessing cycle was repeated 3 times (R1-R3), and its influence on the complex characterization of the TPE-based MREs was thoroughly investigated.

\subsection{Spectral analysis of degradation processes}

The degree of the TPE degradation was monitored after each reprocessing cycle by means of Fourier-transform infra-red (FTIR) spectroscopy. An FTIR instrument Nicolet 6700 (Nicolet, USA) equipped with an ATR-accessory with a diamond crystal was used in order to collect the spectra in a typical wavenumber range of $4000-700 \mathrm{~cm}^{-1}$. The region from 2300 to $1800 \mathrm{~cm}^{-1}$ is not further presented due to the intrinsic absorption of the diamond crystal. The data collection was performed in laboratory conditions, while the spectra resulted from 64 scans with a spectral resolution of $2 \mathrm{~cm}^{-1}$. The TPE degradation was studied also quantitatively via matrix coloration expressed as the yellowness index (YI). This test is standardized according to DIN 6167, which describes the yellowing of near-white or near-colorless materials. For this purpose, a calibrated Color i5 spectrophotometer (X-Rite, Michigan, USA) with the middle area of view aperture was used in the transmission mode at laboratory conditions. The discoid TPE samples with a thickness of $1.25 \mathrm{~mm}$ were characterized from both sides, while 5 readings were taken from each side.

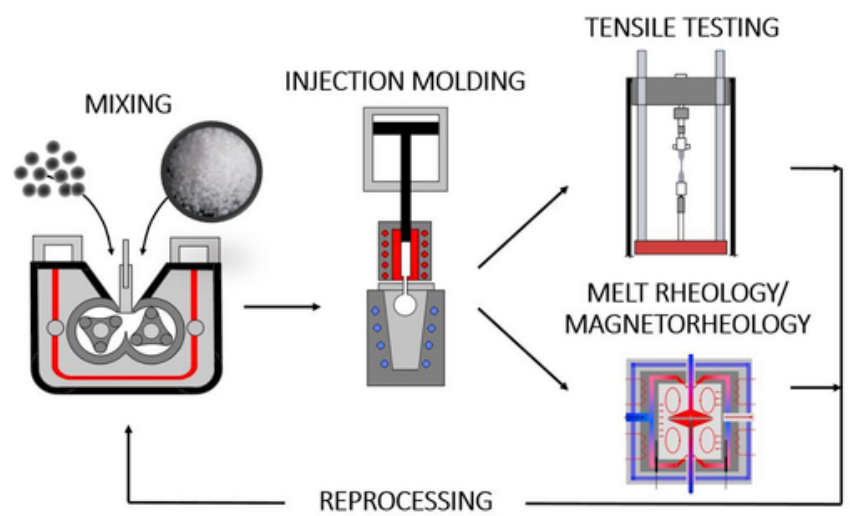

Fig. 1. Scheme illustrating reprocessing cycle of the TPEs, and their MREs. 


\subsection{Molecular weight analysis}

A gel permeation chromatography (GPC) analysis was conducted with a HT-GPC 220 chromatographic system (Agilent, Japan) equipped with a refractive index detector. The samples were dissolved in THF $\left(\sim 3 \mathrm{mg} \mathrm{ml}^{-1}\right.$ of polymer equivalent) at $50{ }^{\circ} \mathrm{C}$ for $4 \mathrm{~h}$. The separation and detection took place on PL gel-mixed bed columns (1x Mixed-A, $300 \times 7.8 \mathrm{~mm}, 15 \mu \mathrm{m}$ particles $+1 \mathrm{x}$ Mixed-B, $300 \times 7.8 \mathrm{~mm}, 10 \mu \mathrm{m}$ particles $+1 \mathrm{x}$ Mixed-D, $300 \times 7.8 \mathrm{~mm}, 5 \mu \mathrm{m}$ particles) at $40{ }^{\circ} \mathrm{C}$ in THF; while its flow rate and the injection volume were set to $1.0 \mathrm{ml} \mathrm{min} \mathrm{m}^{-1}$ and $100 \mu \mathrm{L}$, respectively. The GPC system was calibrated through relative calibration with narrow polystyrene standards ranging from 580 to $271000 \mathrm{~g} \mathrm{~mol}^{-1}$ (Polymer Laboratories Ltd., UK). The weight-average molar mass $\left(\bar{M}_{w}\right)$, number-average molar mass $\left(\bar{M}_{n}\right)$, and the dispersity index $\left(\nexists=\bar{M}_{w} / \bar{M}_{n}\right)$ of the tested samples were determined from peaks corresponding to the relevant polymer fractions. All of the data was processed in Cirrus software.

\subsection{Melt rheology}

Melt rheological experiments were performed on an advanced modular rheometer Physica MCR502 (Anton Paar, Austria) interconnected with a TC 30 temperature control unit with a CTD600 heating chamber. A parallel-plate measuring system with a diameter of $25 \mathrm{~mm}$ (PP25) was used, while the plate gap was set to $1.25 \mathrm{~mm}$. All of the measurements were performed at the processing temperature during the TPEs' and their MREs' fabrication, i.e., $185^{\circ} \mathrm{C}$. The gentle flow of nitrogen was used to limit the thermal oxidation of the TPEs during the data acquisition (heating of $200 \mathrm{~L}_{\mathrm{N}} / \mathrm{h}$, and cooling of $1.0 \mathrm{~m}_{\mathrm{N}}^{3} / \mathrm{h}$ ). The frequency sweeps were performed using angular frequency ranging from $10^{-1}$ to $3 \times 10^{2} \mathrm{rad} \mathrm{s}^{-1}$. In all cases the linearity of the sample response was verified.

\subsection{Microstructure analysis}

A scanning electron microscope (SEM) Nova NanoSEM 450 (FEI, Japan) was used to study the microstructural characteristics of the MREs. A particular emphasis was paid to the effect of reprocessing on particle distribution within TPE matrix. The analysis was performed on cryogenically-fractured $(77 \mathrm{~K})$ samples using the low vacuum detector.

\subsection{Mechanical testing}

The tensile properties of the TPEs, and their MREs were examined using a tensile testing machine M350-5 CT (Testometric Company, Lancashire, UK) at a cross-head speed of $500 \mathrm{~mm} \mathrm{~min}^{-1}$ on the IM samples (specified above). The testing was performed according to the ASTM D638 standard using ten samples in the form of tensile bars (Type V). To process the data, the statistical analysis which included the elimination of outliers (Dean-Dixon test, Q-test; confidence level $\alpha=0.95$ ) was performed. The results were further presented as the arithmetic mean, and the standard deviation.

\subsection{Magnetorheology}

The MR properties of the TPE-based MREs were investigated using a rotational rheometer Physica (MCR502, Anton Paar, Austria) equipped with a magneto-device (MRD 180/1T), and parallel-plate geometry (PP20/MRD/TI/S). The IM discoid sample with a height of $1.25 \mathrm{~mm}$ was placed between the plates, and subjected to oscillatory shear. The possible sample slippage was efficiently reduced by using the sand-blasted upper-plate geometry and applying the constant clamping force of $0.3 \mathrm{~N}$ (perpendicular to the sample). The linear vis- coelasticity region (LVR) was revealed via amplitude-sweeps with the amplitude strain ranging from $10^{-3}-10^{0} \%$ at a frequency of $5 \mathrm{~Hz}$. Further, frequency-sweeps in a range from $10^{-1}-10^{2} \mathrm{~Hz}$ were performed (ensured LVR). The data from both sweep characterizations were collected in the absence of a magnetic field (off-state) as well as under external magnetic field strengths up to $288 \mathrm{kAm}^{-1}$ (on-state). Three independent measurements were performed, and the calculated average values are presented below. The temperature of $25^{\circ} \mathrm{C}$ was kept constant with the help of a heating/cooling device Julabo (FS 18, Germany) during the examinations.

\section{Results and discussion}

The principal advantage of TPEs over conventional vulcanizated elastomers is the possibility to reprocess them. This procedure is accompanied by the recurrent transformation of the TPEs from an elastic into a molten state by conductive and shear heating. As a result, the imposed thermal as well as mechanical stress may lead to the degradation of the TPE matrix [30]. Being aware of these undesirable processes, the degradation of neat TPE matrix was studied as it may further affect the properties of its composites, i.e. the MREs.

\subsection{Degradation of TPE matrix}

The degradation of the TPE matrix was evaluated from the FTIR spectra after each reprocessing cycle. As indicated above, the thermal and mechanical stress increases the vibrations of atoms, and hinders the molecular rotations, which consequently leads to the polymer chain-scission and formation of free radicals. Under common processing conditions the radicals recombine with the present oxygen [31], thus it is reasonable to expect an increased amount of hydroxyl and carbonyl groups in the oxidized TPE. As shown in Fig. 2, a broad peak occurring around $3300 \mathrm{~cm}^{-1}$ clearly indicates the presence of former groups [32], while changes in absorption levels around $1650 \mathrm{~cm}^{-1}$ were assigned to the latter ones [33]. The presence of oxygen-containing groups was generally minimal in the case of the original material, however the indicated absorption peaks gradually increased as the TPEs were introduced to reprocessing.

The processing-induced changes of the TPEs were also studied via YI measurements. Fig. 3 shows the relation between the YI values and the aging time expressed by the reprocessing number (R0-R3). As ap-

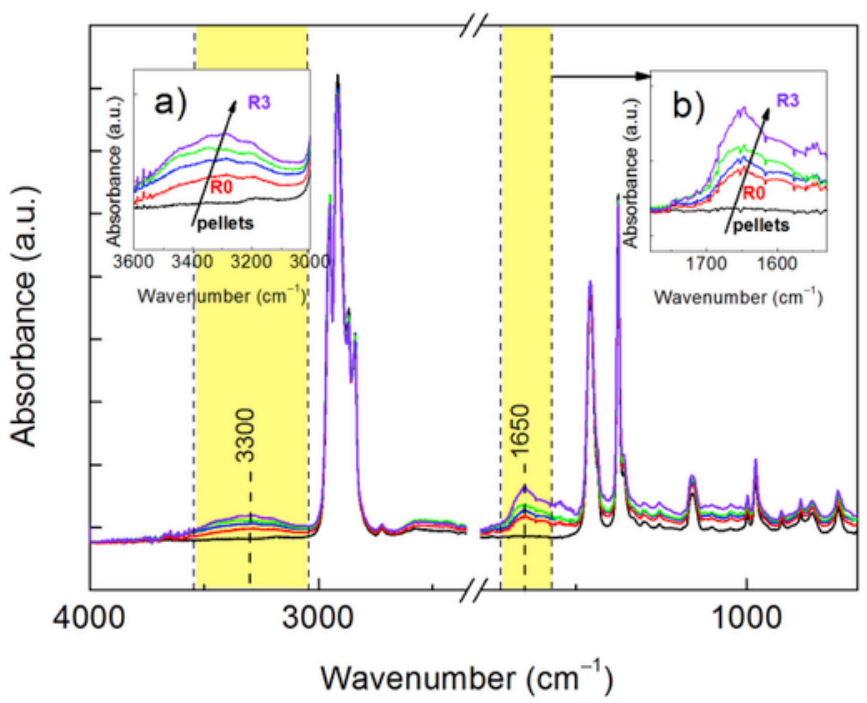

Fig. 2. The FTIR absorption spectra of the original TPE pellets and neat TPE matrices af ter each processing cycle. The insets magnify the regions of $(a)$ hydroxyl and (b) carbonyl group absorptions, respectively. The arrows indicate the processing cycle (R0-R3). 


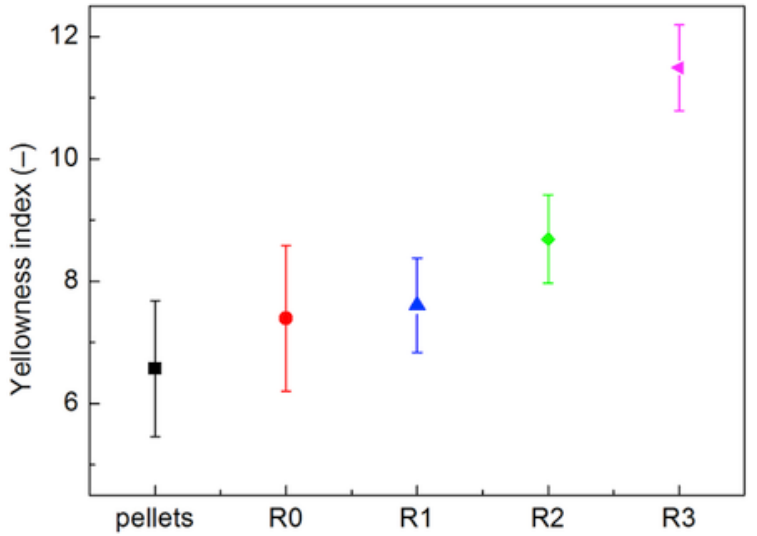

Fig. 3. The YI values of the original TPE pellets and neat TPE matrices after each processing cycle (R0-R3).

parent, the YI values gradually increased reaching almost two times higher in value for the neat TPE matrix after the last processing cycle. It should be mentioned, that the optical properties of the MREs were omitted due to a high particle content. In the case of the TPE matrix, this technique nevertheless served as a complementary tool to delineate the processing-induced matrix degradation.

The changes in molecular weight of the TPE matrices, and their MREs were investigated via GPC, while the original TPE pellets served as a reference. The detailed results are outlined in Table 1. As clearly shown, the $\bar{M}_{n}$ exhibited a decreasing tendency with a number of processing cycles in both material systems, the TPE matrices as well as in their MREs. In more details, an insignificant increase of the $\bar{M}_{n}$ can be noticed on the transition to R1, however it subsequently drops on the transition to the following processing cycles (R2, R3), which may indicate the presence of two competing processes. The first process is the linking of the individual polymer chains, while the second one is the polymer chain-scission into the lower molecular mass fragments. As for the degradation of the other polymers [34], the former process slows down over time, i.e. with the processing number due to a larger mass of recombined chains. As a result, the cleaving of lower-molecular mass fragments prevails over the recombination, which explains the overall $\bar{M}_{n}$ decrease.

Although the trend in the $\bar{M}_{n}$ decrease of the neat TPE matrices and their MREs is similar, in absolute values, a slight difference can be distinguished. While the neat TPE matrix exhibited the $\bar{M}_{n}$ decrease of $\sim 14 \%$ after the last processing cycle, when the MRE variant was calculated, the $\bar{M}_{n}$ decreased by only $\sim 9 \%$. Thus, it can be suggested that the incorporation of the CI particles remarkably reduced the TPE degradation rate. This phenomenon can be explained on the basis of a depth degradation profile, which is typical for polymers [35]. Obviously, a higher oxidation rate is near the material surface, while oxygen starvation takes place in the sample interior. The diffusion of oxy-

Table 1

The GPC data for the original TPE pellets, neat TPE matrices and their MREs after each processing cycle (R0-R3).

\begin{tabular}{lllll}
\hline \multirow{2}{*}{ Material } & Reprocessing cycle $(-)$ & $\begin{array}{l}\overline{\boldsymbol{M}}_{\boldsymbol{n}}\left(\mathrm{g} \cdot \mathrm{mol}^{-1}\right) \\
\times 1000\end{array}$ & $\begin{array}{l}\overline{\boldsymbol{M}}_{\mathrm{w}}\left(\mathrm{g} \cdot \mathrm{mol}^{-1}\right) \\
\times 1000\end{array}$ & $D(-)$ \\
\hline TPE pellets & N/D & 93 & 194 & 2.09 \\
Matrix & R0 & 87 & 191 & 2.20 \\
& R1 & 90 & 192 & 2.13 \\
& R2 & 79 & 179 & 2.27 \\
\multirow{2}{*}{ MRE } & R3 & 80 & 165 & 2.06 \\
& R0 & 90 & 192 & 2.13 \\
& R1 & 91 & 191 & 2.10 \\
& R2 & 86 & 184 & 2.14 \\
& R3 & 85 & 183 & 2.15 \\
\hline
\end{tabular}

gen is therefore the key process defining the extent of the degradation [35]. In the MRE melts, the CI particles represented the barriers across the diffusion paths of oxygen molecules, which is most probably the reason for their less pronounced degradation.

As it is known, the exposure of CI particles to higher temperatures in the presence of air may lead to the formation of less magnetic products such as $\mathrm{FeO}, \mathrm{Fe}_{3} \mathrm{O}_{4}$ and $\mathrm{Fe}_{2} \mathrm{O}_{3}$. However, according to the literature [36], the onset of this process usually occurs at temperatures higher than which were used during the MRE fabrication. The degradation of the CI particles was therefore considered as negligible and all of the changes induced by the reprocessing were attributed solely to the TPE matrix.

\subsection{Melt rheology}

The processing of polymer materials can induce their structural changes on a molecular level, including variations in molecular weight distribution, cross-linking, and/or the formation of low molecular weight fractions [37]. To further analyze whether these changes occurred during the TPE reprocessing, their melt behavior was investigated, by subjecting the TPE melts to oscillatory shear stress. Due to the viscoelastic character of polymer melts, the stress and strain are not in phase [38]. Such a response is usually represented as a complex quantity, widely-known as a complex viscosity, $\eta^{*}$. The mathematical formulation of $\eta^{*}$ is following (Eq. (1)):

$\eta^{*}(\omega)=\eta^{\prime}-i \eta^{\prime \prime}$

where $\eta^{\prime}$ is the in-phase real part, while $\eta^{\prime \prime}$ is the imaginary out-of-phase viscosity, and $i$ is the imaginary number $\left(i^{2}=-1\right)$. To process the complex data, the empirical Cole-Cole model was applied. Although this model was originally introduced in the context of describing the dielectric relaxation spectra of low molecular weight liquids [39], its use was later extended to describe the $\eta^{*}$ data of polymer melts [40] yielding to following equation (Eq. (2)):

$$
\eta^{*}(\omega)=\frac{\eta_{0}}{1+\left(i \omega \lambda_{0}\right)^{1-h}}
$$

where $\eta_{0}$ denotes the zero-shear viscosity, $\omega$ is the angular frequency, $\lambda_{0}$ is the average relaxation time, and $h$ denotes the parameter of the relaxation-time distribution, while $i$ is defined as above. In the complex plane this model typically describes the dependence of $\eta^{\prime \prime}$ on $\eta^{\prime}$ as the arc of a circle. From this data representation $\eta_{0}$ can be determined through the extrapolation of the arc of the circle on the real axis, while the $h$ parameter is represented by the angle between the $\eta^{\prime}$ axis and the radius going from the origin of the axis to the center of the arc of the circle [41].

As can be seen in Fig. 4, the application of the Cole-Cole model generally yielded a good agreement with the experimental data. Based on the results, it can be noticed that the processing time represented by a reprocessing cycle number had a remarkable effect on the properties of the TPE. The numerical results calculated using the Cole-Cole model are summarized in Table 2, where the evolution of $\eta_{0}, \lambda_{0}$, and $h$ fitting values are monitored. Following the reprocessing, the first-mentioned parameter gradually decreased due to the chain-scission of the TPE chains into lower-molecular weight fragments, which corresponds with the GPC results (Table 1). According to Fox and Flory [42], a certain relation between the $\eta_{0}$ and the $\bar{M}_{W}$ exists for linear polymers, which applies, as later shown, also for melts and concentrated solutions of variety of flexible polymers [43]. These quantities are directly proportional for low- $\bar{M}_{W}$ polymers, in which the entanglements between the polymer chains are unlikely or even impossible. However, beyond a certain threshold, termed as the critical molecular weight, $\bar{M}_{C}$, the entanglement coupling between the polymers becomes possible and the 


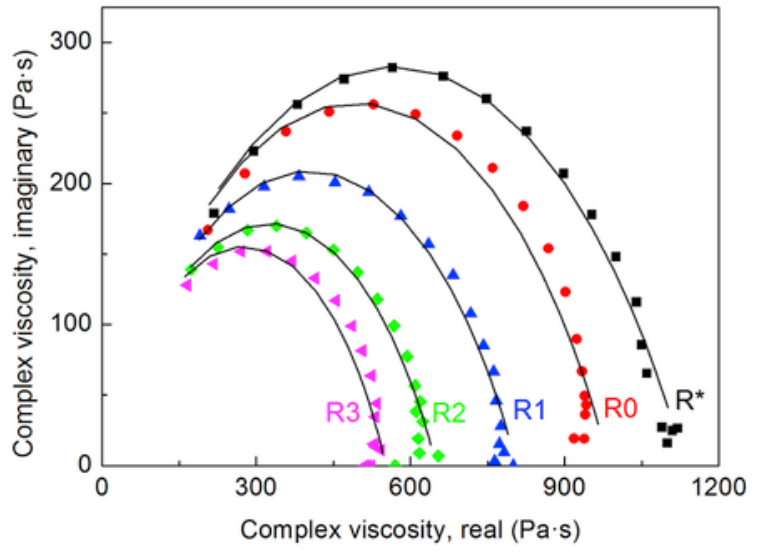

Fig. 4. The Cole-Cole plots for the original TPE pellets $\left(\mathrm{R}^{*}\right)$ and neat TPE matrices after each processing cycle (R0-R3). The solid lines represent the best model fits. The symbol $\mathrm{R}^{*}$ denotes the material processed directly via IM technique (compounding omitted).

Table 2

The Cole-Cole model parameters for the original TPE pellets and neat TPE matrices after each processing cycle (R0-R3).

\begin{tabular}{lllll}
\hline Material & Reprocessing cycle $(-)$ & $\eta_{0}(\mathrm{~Pa} \cdot \mathrm{s})$ & $\lambda_{0}(\mathrm{~s})$ & $h(-)$ \\
\hline TPE pellets & N/D & 1134 & 0.0226 & 0.42 \\
Matrix & R0 & 987.2 & 0.0188 & 0.39 \\
& R1 & 806.5 & 0.0158 & 0.39 \\
& R2 & 650.1 & 0.0123 & 0.38 \\
& R3 & 552.0 & 0.0095 & 0.35 \\
\hline
\end{tabular}

$\eta_{0}$ follows the $\bar{M}_{W}$ via the power-law relation ( $\propto \bar{M}_{W}^{3.4 \pm 0.2}$ ). Considering high $\bar{M}_{W}$ of the commercial TPE (Table 1), the entry into the regime where $\eta_{0} \propto \bar{M}_{W}{ }^{3.4 \pm 0.2}$ can be expected [44]. By validating our data, we have found that the power-law relation applies also for the degradation of neat TPE upon reprocessing. According to the theory, the fitting exponent yielded 3.27 despite relatively small out-of-trend $\bar{M}_{W}$ increase observed during the first processing cycle (R1).

The degradation phenomenon was also manifested in decreasing the $\lambda_{0}$ values with the reprocessing number. However, at a low frequency region, the increase of Newtonian viscosity which is typical for polymer gels was not observed, indicating that the recombination processes were most likely minimal, and any obvious three-dimensional network formation was not obtained [37]. As known from the literature [41], the $h$ parameter generally exhibits values of $0.19-0.20$ for polymers with a narrow molecular-weight distribution ( $\nexists$ equaled to $\sim 1.50$ ) such as PMMA. On the contrary, polymers with broad molecular-weight distribution as PP or HDPE were reported to be characterized by $h$ parameter values of $0.40-0.50$. As obvious from Table 2, the results correlate well with the latter group of materials, however their trend does not exactly match with the GPC results. This might be explained as a typical limitation of the Cole-Cole distribution, which is known to fail at the limit of low frequencies [41].

The same analysis was performed on the MREs, and the viscosity data was depicted in Fig. 5 using the $\eta^{*}$ formalism. As clearly seen, the data exhibited dramatically different characteristics, when compared to those of neat TPEs, thus the presence of the CI particles strongly affected the degradation processes. Approaching the Newtonian low-frequency region it was possible to observe a typical "shoulder" indicating the recombination processes and the formation of a 3D network structure. In a view of further experiments, we assume that the physical network between the TPE chains and the CI particles was developed. Following the processing cycles, the slope of the dependences was generally increasing, and finally (sample R3) almost linear variation was obtained typically describing the densification of the network [37]. The

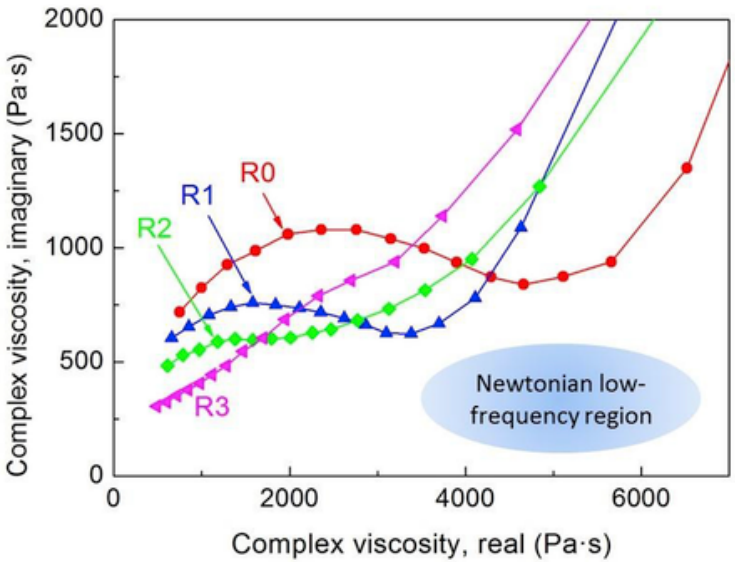

Fig. 5. The Cole-Cole plots for the TPE-based MREs after each processing cycle (R0-R3). The solid lines represent the guide for the eye.

$\eta_{0}$ could not be properly extracted, thus even the Fox and Flory relation [42] was not applied. To conclude, the melt rheological experiments proved the thermo-mechanical degradation of neat TPEs via chain-scission mechanism, while the recombination processes were typical for their MREs. The level of degradation correlated well with the processing number.

\subsection{Microstructure analysis}

The SEM analysis was performed in order to evaluate the effect of reprocessing on the microstructural characteristics of the MREs. Fig. 6 depicts the cross-sectional views of the just-fabricated MRE (R0), and its analogue after the third reprocessing cycle (R3). As can be seen, the CI particles in both systems were well-dispersed without any tendency to agglomerate. Moreover, no air bubbles were observed in the TPE matrix, which is a possible problem of other MREs [45]. These findings suggest a good functional behavior, as the magnetic flux going through the samples is expected to be uniform. Based on the SEM analysis, it appears that the mixing parameters of the TPE-based MREs were suit-
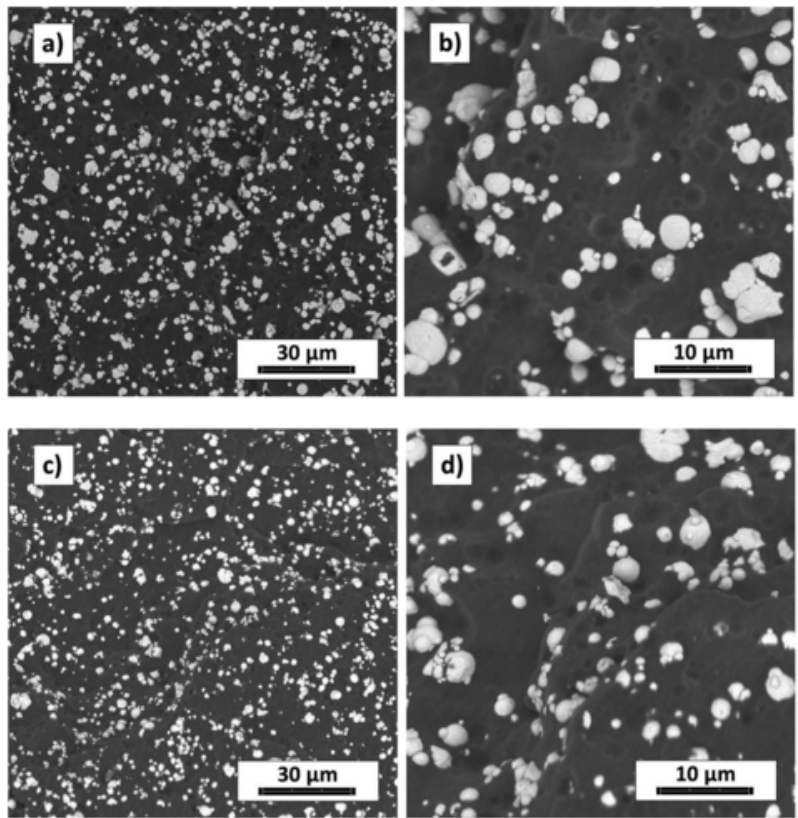

Fig. 6. The SEM images of the isotropic TPE-based MREs, which were just fabricated ( $a$, $b$ ), and after three reprocessing cycles $(c, d)$. 
ably selected, and the reprocessing had an insignificant effect on the particle distribution.

\subsection{Tensile properties}

The tensile properties of neat TPEs and their MREs were investigated after each processing cycle. Fig. 7 displays the representative engineering stress-strain curves. In general, the neat TPEs exhibited slightly higher elongation at the break as well as higher tensile strength values when compared to the MREs' analogues regardless of their processing cycle. This finding indicates that the energy required to break the sample, which is represented by the integral of the individual curves in the stress-strain experiment, was slightly lower after the incorporation of the CI particles. As the CI particles were not surface-treated, their compatibility with the hydrophobic matrices is questionable [10]. The opposite behavior was observed by Rabindranath et al. [46], who functionalized the CI particles with long chains of hydride-terminated polydimethylsiloxane (PDMS). Their particles thus exhibited certain reinforcing properties of the PDMS matrix.

According to the literature [47], the addition of fillers into the TPEs is very different when compared to the situation with the conventional elastomers, such as PDMS. In the TPEs, the reinforcing fillers generally represent the "second" hard phase besides the hard and soft phase already present in the segmented copolymer. Such composites usually exhibit decreased elongation at break, but simultaneously their stiffness is
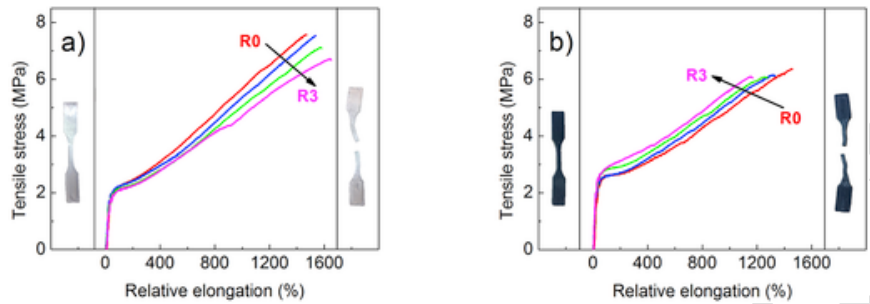

Fig. 7. Representative tensile data for $(a)$ neat TPEs, and $(b)$ their MREs. The arrows indicate the processing cycle (R0-R3).
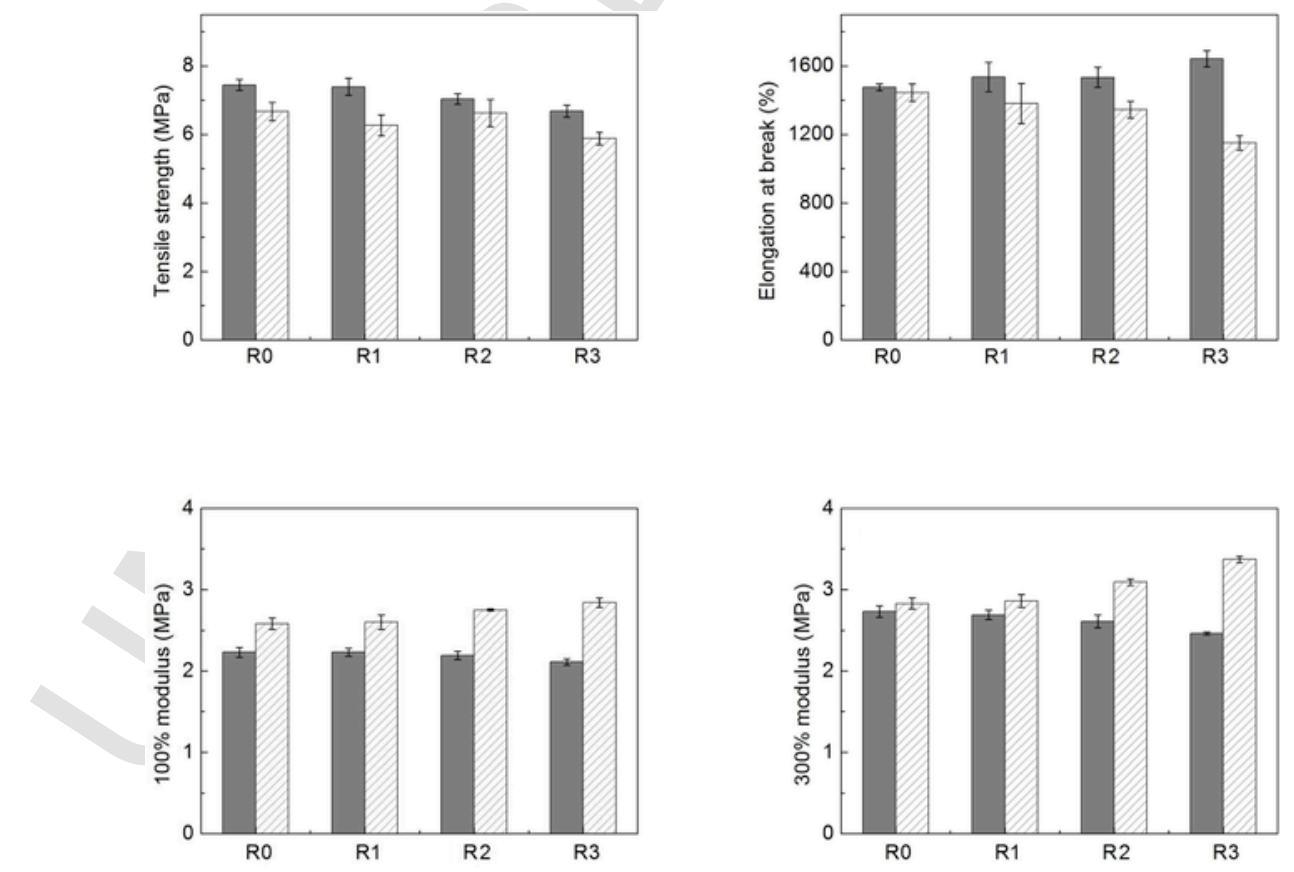

Fig. 8. The mechanical parameters of the neat TPE matrices (dark) and their MREs (stripped) after each processing cycle (R0-R3). evaluation of the systems in the context of reprocessing. $5)$. (Fig. 7, inset). ticle/matrix bonding.

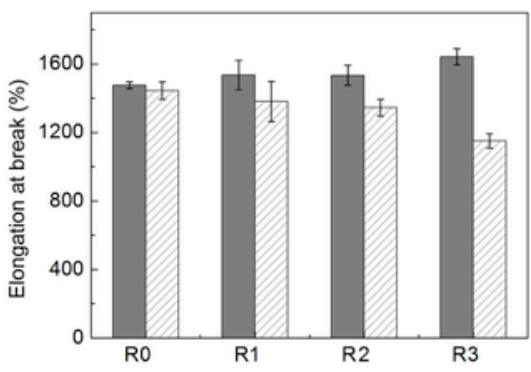

increased [47]. As indicated above, the addition of the CI particles generally followed the trends observed in the literature, and more details will be given further in text. However, at this point let us focus on the

The effect of the number of processing iterations on mechanical quantities, including the tensile modulus at $100 \%$, and $300 \%$ elongation, was evaluated. Based on the results (Figs. 7 and 8), reprocessing influenced the mechanical properties of both the neat TPE matrices and their MREs, however different trends were observed. For neat TPE matrix, the tensile strength decreased by $\sim 10 \%$, and the elongation at the break increased by $\sim 11 \%$ after the last processing cycle. The former change can be explained as a consequence of decreased $\bar{M}_{n}$ (Table 1), while the latter was attributed to the development of scarce transversal cross-links [48]. A similar phenomenon connected to the cross-linking was reported by Li et al. [49], however in their TPEs, the cross-linking was induced by the addition of dicumyl peroxide. It is worth mentioning that in the MREs, the tensile strength remained virtually unchanged, but the elongation at the break decreased by $\sim 20 \%$ after the last processing cycle. We assume that this unusual behavior was caused due to the interfacial particle/matrix bonding [48]. The CI particles are under normal conditions covered by a thin layer of oxides which could potentially react with the free radicals generated during the thermo-mechanical induced TPE chain scission. For the complexity, it should be mentioned that this result is in accordance with the melt rheology experiments (Fig.

As a specifying remark we note, that although the materials exhibited high elongations at break, they recovered upon breaking nearly to the original state exhibiting relatively small permanent deformations

Finally, the modulus of the material can be enhanced by the incorporation of the micro- or nanoparticles because solid particles have a much higher stiffness when compared to that of the matrix [50]. This aspect corresponds to our results. As seen in Fig. 8, the MREs exhibited a slightly enhanced modulus when compared to neat TPEs [47], but more importantly their modulus increased by $\sim 11-19 \%$ after the last processing number, which again supports the theory of processing-induced par- 


\subsection{Magnetorheological activity}

To reveal the proper conditions for the MR testing, the MREs were firstly subjected to the amplitude-sweeps. As seen from Fig. 9, the samples exhibited a typical viscoelastic response; at low strains the storage modulus of the TPE-based MREs was strain-independent, defining the LVR, while at higher strains their storage modulus gradually decreased, suggesting permanent sample deformations [25]. As will be explained further in this text, the storage modulus of the MREs increased after each reprocessing cycle indicating remarkable changes in their shear-mechanical properties. However, neither the reprocessing number nor the applied $H$ had any significant effect on the LVR position (Fig. 9). Thus, the strain value of $10^{-2} \%$ was chosen for all the experiments.

Fig. 10 shows the storage modulus dependences of the recycled TPE-based MREs on the excitation frequency. As seen, the storage modulus increased notably after each reprocessing cycle, thus exhibiting a similar trend as the tensile modulus (Fig. 8). This behavior suggested differences in the MR activity of the recycled MREs. According to the literature [7], the matrix elasticity significantly effects the particle restructuration and consequently the MR effect, which is given according to the equation (Eq. (3)):

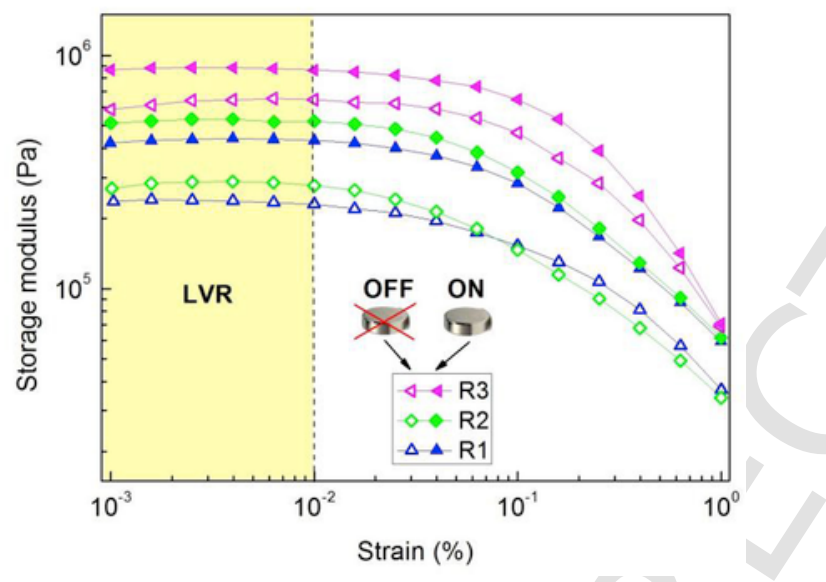

Fig. 9. The off-state (open symbols) and the on-state (solid symbols) storage modulus dependences as the functions of strain for the TPE-based MREs after each reprocessing cycle (R1-R3).

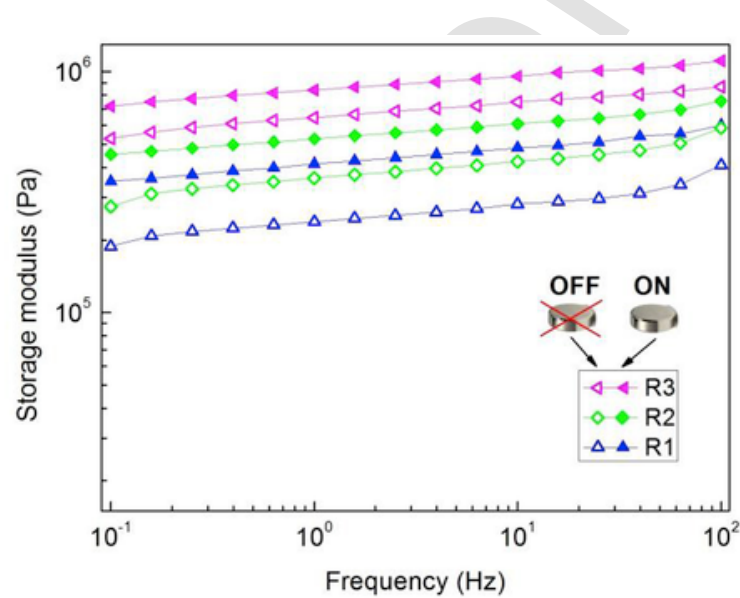

Fig. 10. The off-state (open symbols) and the on-state (solid symbols) storage modulus dependences as functions of frequency for the TPE-based MREs after each reprocessing cycle (R1-R3).
Relative MR effect $=\frac{\left(\mathrm{G}_{\mathrm{H}}^{\prime}-\mathrm{G}_{0}^{\prime}\right)}{\mathrm{G}_{0}^{\prime}} \times 100 \%$

where $G_{H}^{\prime}$, and $G_{0}^{\prime}$ are the field-on, and the field-off storage moduli, respectively. The relative MR effects were quantified under a model situation (frequency of $1 \mathrm{~Hz}$, magnetic field strength of $288 \mathrm{kAm}^{-1}$ ) giving the values of $68 \%, 46 \%$, and $29 \%$ for the recycled samples. This trend can be explained as a consequence of lower relative particle motions within the body of the reprocessed MREs [10]. On a molecular level, the already-mentioned covalent particle/matrix bonding mechanism most probably participated between the free radicals formed during the TPE chain-scission and the hydroxyl groups occurring on the surface of the CI particles. A similar phenomenon was recently observed in the PDMS-based MREs [46,51].

Although the achieving of high MR effects was not a primary goal of this study, it is important to mention that the presented MREs exhibited rather moderate MR activity after reprocessing when compared to the MREs which were based on soft matrices [7,52]. The softening of the matrix can be executed by the incorporation of the plasticizers [52] or by varying the cross-linker amount [11]. Another strategy in enhancing the MR effects is particle grafting with polymers, modifying the particle/matrix interactions [51]. Moreover, in some papers [25,53] high magnetic fields $\left(H>850 \mathrm{kAm}^{-1}\right)$ were applied, which are difficult to achieve in a uniform state using the MRD180/1T magneto-cell [54].

To conclude, we have shown that recyclable TPE-based MREs can be readily fabricated via the IM process, but the reprocessing-induced decrease of their MR effect has to be considered. In order to achieve a lower manufacturing cost and increase the competitiveness of these materials, a different reprocessing method could be adopted similarly as with powder injection molding technology. Instead of reprocessing the whole batch in the following cycles, the reprocessed feedstock can be used only as an addition (30-50\%) to the original materials [55]. This approach could be an effective way to preserve sufficient mechanical properties and the MR effect after several reprocessing cycles.

\section{Conclusions}

In order to overcome the limitations of the conventional MREs based on chemically cross-linked matrices, we adopted the TPE as an alternative matrix material. The TPE-based MREs were fabricated via the IM technique and subjected to three reprocessing cycles. Following the cycles, the content of oxygen-containing groups increased, the $\bar{M}_{n}$ decreased by one tenth, while the YI almost doubled. Melt rheology experiments revealed the thermo-mechanically induced chain-scission in neat TPEs $\left(\eta_{0} \propto \bar{M}_{W}^{3.27}\right)$, while the presence of the CI particles rather provoked the recombination processes manifesting as the particle/matrix bonding. The incorporation of the CI particles slightly enhanced the tensile modulus of the MREs without a significant loss in elasticity when compared to neat TPEs. The unexpected trends in the mechanical behavior of the MREs indicated a side process, which further supported the ongoing particle/matrix bonding phenomenon. As a result, $G_{0}^{\prime}$ of the MREs gradually increased after each processing iteration. Due to decreased particle mobility, the relative MR effect was decreasing by tens of percent per cycle. We expect that the injection-molded TPE-based MREs could offer a new pathway for producing the smart engineering composites with more complicated shapes owing to the ability to be easily reprocessed.

\section{Acknowledgements}

The author M. C. acknowledges the project International Mobility of TBU in Zlín Researchers [No. CZ.02.2.69/0.0/0.0/16_027/0008464] 
funded from the EU Funds - OP Research, Development and Education in cooperation with the Ministry of Education, Youth and Sports, Czech Republic. The authors wish to thank the Czech Science Foundation [17-24730S] for their financial support. This work was also supported by the Ministry of Education, Youth and Sports of the Czech Republic - Program NPU I [LO1504].

\section{References}

[1] K.H. Lam, Z.H. Chen, Y.Q. Ni, H.L.W. Chan, A magnetorheological damper capable of force and displacement sensing, Sens Actuators, A 158 (2010) 51-59, https:// doi.org/10.1016/j.sna.2009.12.022.

[2] S.S. Sun, J. Yang, H.X. Deng, H. Du, W.H. Li, G. Alici, M. Nakano, Horizontal vibration reduction of a seat suspension using negative changing stiffness magnetorheological elastomer isolators, Int. J. Vehicle Des. 68 (2015) 104-118, https:// doi.org/10.1504/ijvd.2015.071076.

[3] M. Behrooz, F. Gordaninejad, A flexible micro fluid transport system featuring magnetorheological elastomer, Smart Mater Struct 25 (2016) https://doi.org/10. 1088/0964-1726/25/2/025011, 025011.

[4] M. Zrinyi, D. Szabo, Muscular contraction mimiced by magnetic gels, Int J Mod Phys B 15 (2001) 557-563, https://doi.org/10.1142/s0217979201005015.

[5] M. Cvek, R. Moucka, M. Sedlacik, V. Babayan, V. Pavlinek, Enhancement of radio-absorbing properties and thermal conductivity of polysiloxane-based magnetorheological elastomers by the alignment of filler particles, Smart Mater Struct 26 (2017) https://doi.org/10.1088/1361-665X/aa7ef6, 095005

[6] R.K. Shuib, K.L. Pickering, B.R. Mace, Dynamic properties of magnetorheological elastomers based on iron sand and natural rubber, J Appl Polym Sci 132 (2015) 41506, https://doi.org/10.1002/app.41506.

[7] A.V. Chertovich, G.V. Stepanov, E.Y. Kramarenko, A.R. Khokhlov, New composite elastomers with giant magnetic response, Macromol Mater Eng 295 (2010) 336-341, https://doi.org/10.1002/mame.200900301.

[8] M. Lokander, T. Reitberger, B. Stenberg, Oxidation of natural rubber-based magnetorheological elastomers, Polym Degrad Stabil 86 (2004) 467-471, https://doi. org/10.1016/j.polymdegradstab.2004.05.019.

[9] Y. Wang, X.L. Gong, J. Yang, S.H. Xuan, Improving the dynamic properties of mre under cyclic loading by incorporating silicon carbide nanoparticles, Ind Eng Chem Res 53 (2014) 3065-3072, https://doi.org/10.1021/ie402395e.

[10] M. Cvek, M. Mrlik, M. Ilcikova, J. Mosnacek, L. Munster, V. Pavlinek, Synthesis of silicone elastomers containing silyl-based polymer grafted carbonyl iron particles: an efficient way to improve magnetorheological, damping, and sensing performances, Macromolecules 50 (2017) 2189-2200, https://doi.org/10.1021/acs. macromo1.6b02041.

[11] Y.C. Fan, X.L. Gong, S.H. Xuan, L.J. Qin, X.F. Li, Effect of cross-link density of the matrix on the damping properties of magnetorheological elastomers, Ind Eng Chem Res 52 (2013) 771-778, https://doi.org/10.1021/ie302536e.

[12] X.L. Gong, Y.C. Fan, S.H. Xuan, Y.G. Xu, C. Peng, Control of the damping properties of magnetorheological elastomers by using polycaprolactone as a temperature-controlling component, Ind Eng Chem Res 51 (2012) 6395-6403, https://doi. org/10.1021/ie300317b.

[13] M. Lokander, B. Stenberg, Improving the magnetorheological effect in isotropic magnetorheological rubber materials, Polym Test 22 (2003) 677-680, https://doi. org/10.1016/s0142-9418(02)00175-7.

[14] A. Boczkowska, S.F. Awietjan, R. Wroblewski, Microstructure-property relationships of urethane magnetorheological elastomers, Smart Mater Struct 16 (2007) 1924-1930, https://doi.org/10.1088/0964-1726/16/5/049.

[15] B.X. Ju, R. Tang, D.Y. Zhang, B.L. Yang, M. Yu, C.R. Liao, X.L. Yuan, L.W. Zhang, J.H. Liu, Dynamic mechanical properties of magnetorheological elastomers based on polyurethane matrix, Polym Compos 37 (2016) 1587-1595, https://doi.org/10 1002/pc. 23330.

[16] Ubaidillah, F. Imaduddin, Y.C. Li, S.A. Mazlan, J. Sutrisno, T. Koga, I. Yahya, S.B. Choi, A new class of magnetorheological elastomers based on waste tire rubber and the characterization of their properties, Smart Mater Struct 25 (2016) 115002, https://doi.org/10.1088/0964-1726/25/11/115002.

[17] S. Datta, K. Naskar, Y.K. Bhardwaj, S. Sabharwal, A study on dynamic rheological characterisation of electron beam crosslinked high vinyl styrene butadiene styrene block copolymer, Polym Bull 66 (2011) 637-647, https://doi.org/10.1007/ s00289-010-0359-x

[18] K.S. O'Connor, A. Watts, T. Vaidya, A.M. LaPointe, M.A. Hillmyer, G.W. Coates, Controlled chain walking for the synthesis of thermoplastic polyolefin elastomers: synthesis, structure, and properties, Macromolecules 49 (2016) 6743-6751, https: //doi.org/10.1021/acs.macromol.6b01567.

[19] P. Malecki, M. Krolewicz, J. Krzak, J. Kaleta, J. Piglowski, Dynamic mechanical analysis of magnetorheological composites containing silica-coated carbonyl iron powder, J Intell Mater Syst Struct 26 (2015) 1899-1905, https://doi.org/10.1177/ 1045389x15581522.

[20] X.Y. Qiao, X.S. Lu, X.L. Gong, T. Yang, K. Sun, X.D. Chen, Effect of carbonyl iron concentration and processing conditions on the structure and properties of the thermoplastic magnetorheological elastomer composites based on poly(styrene-b-ethylene-co-butylene-b-styrene) (SEBS), Polym Test 47 (2015) 51-58, https://doi.org/10.1016/j.polymertesting.2015.08.004.

[21] X.Y. Qiao, X.S. Lu, W.H. Li, J. Chen, X.L. Gong, T. Yang, W. Li, K. Sun, X.D. Chen, Microstructure and magnetorheological properties of the thermoplastic magnetorheological elastomer composites containing modified carbonyl iron particles and poly(styrene-b-ethylene-ethylenepropylene-b-styrene) matrix, Smart Mater Struct 21 (2012) 115028, https://doi.org/10.1088/0964-1726/21/11/115028.
[22] M. Sedlacik, M. Mrlik, V. Babayan, V. Pavlinek, Magnetorheological elastomers with efficient electromagnetic shielding, Compos Struct 135 (2016) 199-204, https://doi.org/10.1016/j.compstruct.2015.09.037.

[23] S.R. Khimi, K.L. Pickering, The effect of silane coupling agent on the dynamic mechanical properties of iron sand/natural rubber magnetorheological elastomers, Composites, Part B 90 (2016) 115-125, https://doi.org/10.1016/j.compositesb. 2015.11.042

[24] S.R. Khimi, K.L. Pickering, B.R. Mace, Dynamic properties of magnetorheological elastomers based on iron sand and natural rubber, J Appl Polym Sci 132 (2015) 41506, https://doi.org/10.1002/app.41506.

[25] V.V. Sorokin, G.V. Stepanov, M. Shamonin, G.J. Monkman, A.R. Khokhlov, E.Y. Kramarenko, Hysteresis of the viscoelastic properties and the normal force in magnetically and mechanically soft magnetoactive elastomers: effects of filler composition, strain amplitude and magnetic field, Polymer 76 (2015) 191-202, https:// doi.org/10.1016/j.polymer.2015.08.040.

[26] M. Cvek, R. Moucka, M. Sedlacik, V. Pavlinek, Electromagnetic, magnetorheological and stability properties of polysiloxane elastomers based on silane-modified carbonyl iron particles with enhanced wettability, Smart Mater Struct 26 (2017) 105003, https://doi.org/10.1088/1361-665X/aa85c5.

[27] V. Volpe, D. Davino, L. Sorrentino, G. Gorrasi, R. Pantani, Smart behavior of elastomeric composites produced by injection molding, J Appl Polym Sci 135 (2018) 7, https://doi.org/10.1002/app.46863.

[28] V. Volpe, M. D'Auria, L. Sorrentino, D. Davino, R. Pantani, Injection molding of magneto-sensitive polymer composites, Mater. Today Commun. 15 (2018) 280-287, https://doi.org/10.1016/j.mtcomm.2018.03.016.

[29] V. Volpe, M. D'Auria, L. Sorrentino, D. Davino, R. Pantani, Magneto-mechanical behavior of elastomeric carbonyl iron particles composite foams produced by foam injection molding, J Magn Magn Mater 466 (2018) 44-54, https://doi.org/10. 1016/j.jmmm.2018.06.071.

[30] L. Behalek, J. Habr, M. Seidl, P. Lenfeld, M. Boruvka, Thermal degradation of the thermoplastic elastomers during the injection moulding process, Mater Sci Forum 862 (2016) 148-155.

[31] D. Schmiederer, A. Gardocki, I. Kuhnert, E. Schmachtenberg, Local thermo-oxidative degradation in injection molding, Polym Eng Sci 48 (2008) 717-722, https:// doi.org/10.1002/pen.21011.

[32] C.L. de Carvalho, A.F. Silveira, D.D. Rosa, A study of the controlled degradation of polypropylene containing pro-oxidant agents, SpringerPlus 2 (2013) 623, https:// doi.org/10.1186/2193-1801-2-623.

[33] C. Kosa, M. Sedlacik, A. Fiedlerova, S. Chmela, K. Borska, J. Mosnacek, Photochemically cross-linked poly(epsilon-caprolactone) with accelerated hydrolytic degradation, Eur Polym J 68 (2015) 601-608, https://doi.org/10.1016/j. eurpolymj.2015.03.041.

[34] L. Munster, J. Vicha, J. Klofac, M. Masar, P. Kucharczyk, I. Kuritka, Stability and aging of solubilized dialdehyde cellulose, Cellulose 24 (2017) 2753-2766, https:// doi.org/10.1007/s10570-017-1314-x.

[35] M. Zanetti, P. Bracco, L. Costa, Thermal degradation behaviour of PE/clay nanocomposites, Polym Degrad Stabil 85 (2004) 657-665, https://doi.org/10. 1016/j.polymdegradstab.2004.03.005.

[36] M.A. Abshinova, I. Kuritka, N.E. Kazantseva, J. Vilcakova, P. Saha, Thermomagnetic stability and heat-resistance properties of carbonyl iron filled siloxanes, Mater Chem Phys 114 (2009) 78-89, https://doi.org/10.1016/j.matchemphys. 2008.08.091

[37] P. Stloukal, V. Verney, S. Commereuc, J. Rychly, L. Matisova-Rychla, V. Pis, M. Koutny, Assessment of the interrelation between photooxidation and biodegradation of selected polyesters after artificial weathering, Chemosphere 88 (2012) 1214-1219, https://doi.org/10.1016/j.chemosphere.2012.03.072.

[38] R.B. Bird, A.J. Giacomin, Who conceived the "complex viscosity"?, Rheol Acta 51 (2012) 481-486, https://doi.org/10.1007/s00397-012-0621-2.

[39] K.S. Cole, R.H. Cole, Dispersion and absorption in dielectrics: I. Alternating current characteristics, J Chem Phys 9 (1941) 341-351.

[40] C.A. Garcia-Franco, D.W. Mead, Rheological and molecular characterization of linear backbone flexible polymers with the Cole-Cole model relaxation spectrum, Rheol Acta 38 (1999) 34-47, https://doi.org/10.1007/s003970050154.

[41] V. Verney, A. Michel, Representation of the rheological properties of polymer melts in terms of complex fluidity, Rheol Acta 28 (1989) 54-60, https://doi.org/ $10.1007 /$ bf01354769.

[42] T.G. Fox, P.J. Flory, Further studies on the melt viscosity of polyisobutylene, J Phys Colloid Chem 55 (1951) 221-234, https://doi.org/10.1021/j150485a010.

[43] R.H. Colby, L.J. Fetters, W.W. Graessley, Melt viscosity molecular-weight relationship for linear-polymers, Macromolecules 20 (1987) 2226-2237, https://doi.org/ 10.1021/ma00175a030

[44] L.J. Fetters, D.J. Lohse, S.T. Milner, W.W. Graessley, Packing length influence in linear polymer melts on the entanglement, critical, and reptation molecular weights, Macromolecules 32 (1999) 6847-6851, https://doi.org/10.1021/ ma990620o.

[45] C.L. Lian, K.H. Lee, C.H. Lee, Friction and wear characteristics of magneto-rheolog ical elastomers based on silicone/polyurethane hybrid, J Tribol, Trans Asme 137 (2015)031607https://doi.org/10.1115/1.4029942.

[46] R. Rabindranath, H. Bose, On the mobility of iron particles embedded in elastomeric silicone matrix, In: H.I. Unal (Ed.), 13th int. Conf. Electrorheol. Fluids magnetorheol. Susp, vol. 412, Iop Publishing Ltd, Bristol, 2013.

[47] E. Wisse, L.E. Govaert, H.E.H. Meijer, E.W. Meijer, Unusual tuning of mechanical properties of thermoplastic elastomers using supramolecular fillers, Macromolecules 39 (2006) 7425-7432, https://doi.org/10.1021/ma060986i.

[48] O.P. Grigoryeva, A.M. Fainleb, V.F. Shumskii, V.A. Vilenskii, N.V. Kozak, N.V. Babkina, The Effect of multi-reprocessing on the structure and characteristics of thermoplastic elastomers based on recycled polymers, Polym Sci 51 (2009) 216-225, https://doi.org/10.1134/s0965545x09020114. 
[49] Y. Li, Y. Zhang, Y.X. Zhang, Structure and mechanical properties of SRP/HDPE/ POE (EPR or EPDM) composites, Polym Test 22 (2003) 859-865, https://doi.org/ 10.1016/s0142-9418(03)00022-9.

[50] S.Y. Fu, X.Q. Feng, B. Lauke, Y.W. Mai, Effects of particle size, particle/matrix interface adhesion and particle loading on mechanical properties of particulate-polymer composites, Composites, Part B 39 (2008) 933-961, https://doi.org/10.1016/ j.compositesb.2008.01.002.

[51] M. Cvek, M. Mrlik, J. Sevcik, M. Sedlacik, Tailoring performance, damping, and surface properties of magnetorheological elastomers via particle-grafting technology, Polymers 10 (2018) 1411.

[52] A. Stoll, M. Mayer, G.J. Monkman, M. Shamonin, Evaluation of highly compliant magneto-active elastomers with colossal magnetorheological response, J Appl Polym Sci 131 (2014) 39793, https://doi.org/10.1002/app.39793.
[53] G.J. Liao, Y.G. Xu, F.J. Wang, F.Y. Wei, Q. Wan, Influence of gamma radiation on the shear modulus of magnetorheological elastomer, Mater Lett 174 (2016) 79-81, https://doi.org/10.1016/j.matlet.2016.03.085.

[54] H.M. Laun, G. Schmidt, C. Gabriel, C. Kieburg, Reliable plate-plate MRF magnetorheometry based on validated radial magnetic flux density profile simulations, Rheol Acta 47 (2008) 1049-1059, https://doi.org/10.1007/s00397-008-0305-0.

[55] L.H. Cheng, K.S. Hwang, Y.L. Fan, Molding properties and causes of deterioration of recycled powder injection molding feedstock, Metall Mater Trans A 40A (2009) 3210-3216, https://doi.org/10.1007/s11661-009-0071-3. 